\title{
Influence of Solvent on Photoinduced Electron-Transfer Reaction: Time-Resolved Resonance Raman Study
}

\author{
Himansu Mohapatra and Siva Umapathy* \\ Department of Inorganic and Physical Chemistry, Indian Institute of Science, Bangalore-560012, India
}

Received: April 26, 2009

\begin{abstract}
Time-resolved resonance Raman spectroscopy (TR3) has been used to study the effect of solvent polarity on the mechanism and nature of intermediates formed in photoinduced electron-transfer reaction between triplet flouranil $\left({ }^{3} \mathrm{FL}\right)$ and tetramethylbenzene (TMB). Comparison of the TR3 spectra in polar, nonpolar, and medium polar media suggests that formation of radical anion due to electron-transfer reaction between ${ }^{3} \mathrm{FL}$ and TMB is favored in more polar solvents, whereas ketyl radical formation is more favored in less polar media. Compared to ketyl radical, the extent of radical anion formation is negligible in nonpolar solvents. Therefore, it is inferred that in nonpolar media ketyl radical is mainly generated by hydrogen-transfer reaction in the encounter complex between ${ }^{3} \mathrm{FL}$ and TMB. In solvents of medium polarity, the ion-pair decay leads to the formation of both ketyl radical and ketyl radical formed from the encounter between triplet state and the donor. Thus, competition between the formation of ketyl radical and ion pair is influenced by the solvent polarity. The nature of the ion pair in different solvent polarity has been investigated from the changes observed in the vibrational frequency of (fluoranil) FL part of the complex.
\end{abstract}

\section{Introduction}

Photoinduced electron-transfer reactions have received much attention in recent years, with a view to investigate the oxidation and reduction mechanism of molecules in the excited state. Since most electron-transfer reactions take place in condensed media, the influence of medium is of great importance for understanding the mechanism and nature of intermediates formed in the reaction. The ion pair formed between a donor and acceptor has been recognized as a key intermediate in photoinduced electron-transfer reactions. ${ }^{1}$ Competition between the stabilization gained from Coulombic interaction of the ions in an ion pair and the solvation of the ions has important consequence on the nature of the ion pair, which can be either a contact ion pair (CIP) or a solvent-separated ion pair (SSIP). In this respect, solvent polarity has revealed interesting features in the distribution of these intermediates. ${ }^{2-8}$ In polar solvents, triplet ion pairs, commonly observable in the nanosecond time domain from bimolecular photoinduced electron-transfer reactions, are mainly SSIP, whereas in less polar solvents CIPs are expected in the same timescale. ${ }^{9-11}$ In highly polar solvents such as acetonitrile, the SSIP may dissociate into solvated ions. Transient absorption studies have shown that, in nonpolar media, CIP between chloranil and acenaphthene decays through intra-ion-pair proton transfer and, in polar solvents, the nature of ion pair is SSIP, which favors ionic dissociation into free anion and cation radicals. ${ }^{9}$ Chloranil durene system in 1,2-dichloromethane revealed the presence of both CIP and SSIP in equilibrium. ${ }^{10}$ But in such studies reported thus far, there is no structural evidence to differentiate the nature of ion pair formed in different solvent polarity.

As the polarity of solvent decreases, the structure of CIP becomes less ionic because of mixing of the locally excited triplet state and the ionic CT state. Therefore, CIP can be differentiated from SSIP or solvated ions in terms of donor-accep-

* To whom correspondence should be addressed. Telephone: +91-8023601234. Fax: +91-80-23601552. E-mail: umapathy@ipc.iisc.ernet.in.

\section{SCHEME 1}
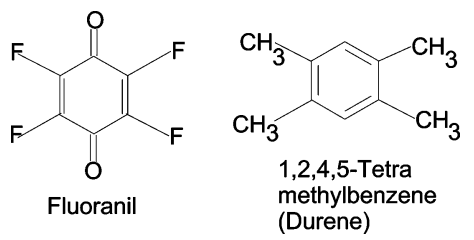

tor interaction and extent of charge transfer in the CIP. This kind of species in which the extent of charge transfer is less compared to the pure ionic $\mathrm{CT}$ state is referred to as exciplex. Therefore, probing the structural difference between CIP (exciplex) and SSIP will give good insight into the nature of the ion pair formed in different solvent polarity. In this work, TR3 spectroscopy was used to study the effect of solvent polarity on the nature of the ion pair and the mechanism of photoinduced electron-transfer reaction. The solvent polarity is varied by using a mixture of acetonitrile $\left(\mathrm{CH}_{3} \mathrm{CN}\right)$ and carbon tetrachloride $\left(\mathrm{CCl}_{4}\right)$ in different proportions. The systems employed for the study are fluoranil (FL) as the electron acceptor and tetramethylbenzene (Durene) (TMB) as electron donor. The structures of FL and TMB are shown in Scheme 1. In FL, the flourine atom induces strong electron withdrawing (by inductive effect) and a weak electron pair donating ( $\pi$ bonding) effect, which is referred to as the perfluoro effect. ${ }^{12}$ The presence of such effects will significantly affect the mechanism of photoinduced electrontransfer reactions compared to other halogenated quinones. We previously reported ${ }^{13,14}$ detailed TR3 studies on triplet fluoranil $\left({ }^{3} \mathrm{FL}\right)$, where we investigated the influence of perfluorination on its structure and reactivity.

TR3 spectroscopy is an excellent technique for probing the structure of the molecule in the excited state. This technique has been used widely to study the structure and dynamics of intermediates formed in photoinduced electron-transfer reaction. ${ }^{15-17}$ For example, TR3 studies on 9,10-anthraquinone-1,2,4trimethoxybenzene revealed the presence of geminate ion pair 
and its separation into free ions in photoinduced electron-transfer reaction. The TR3 spectra show shifting of the band position of 1,2,4-trimethoxybenzene cation radical as the ion pair proceeds toward separation. ${ }^{15,16}$ Further, using this method, Tahara and Hamaguchi extensively investigated structural evidence on the exciplex nature of the ion pair in the case of $p$-chloranil alkylbenzene charge-transfer complex. ${ }^{17}$ Our main objectives of this study are (a) to understand influence of medium on the mechanism of formation and decay of ion pair and (b) to provide structural evidence on the nature of the ion pair formed in different solvent polarities using TR3 spectroscopy.

\section{Experimental Section}

The experimental apparatus and procedures used for the TR3 spectroscopy were described in detail previously. ${ }^{18}$ Briefly, the photoexcitation pump wavelength is the third-harmonic 355nm output of a Nd:YAG laser (DCR-11), and the probe wavelength $416 \mathrm{~nm}$ is from a homemade $\mathrm{H}_{2}$ Raman shifter. The laser pulses are about 8-10 ns in temporal width, and energies are about 1.0 and $0.5 \mathrm{~mJ}$ for pump and probe, respectively. The delay between the laser pulses is provided by the standard DG535 delay generator. A SPEX 1404 double monochromator was used with two 600 grooves grating to disperse the scattered light. A liquid nitrogen-cooled CCD (Princeton instrument) with $576 \times 376$ pixel was used as a multichannel detector. TMB and $\mathrm{FL}$ were obtained from Aldrich. The $\mathrm{CH}_{3} \mathrm{CN}$ and $\mathrm{CCl}_{4}$ used were of analytical grade and distilled before use. The recorded Raman spectra were calibrated using known solvent bands as reference, and the spectral resolution was estimated as $5 \mathrm{~cm}^{-1}$.

In transient absorption measurement, the laser excitation pulse at $355 \mathrm{~nm}$ was obtained from the Nd:YAG laser. The sample cell used had a path length of $1 \mathrm{~cm}$, and the signals were detected using a $250 \mathrm{~W}$ pulsed xenon lamp, Czerny Turner monochromator, and R-928 photomultiplier tube. The signals were acquired in an oscilloscope and then processed.

\section{Results and Discussion}

Transient Absorption Studies. Photoinduced electrontransfer reaction between FL and TMB was studied under varying solvent polarity conditions. The polarity was varied by using a mixture of $\mathrm{CH}_{3} \mathrm{CN}$ and $\mathrm{CCl}_{4}$ in different proportions. Transient absorption spectra recorded from solvent mixture (a) 4:1 (v/v) $\mathrm{CCl}_{4}-\mathrm{CH}_{3} \mathrm{CN}$, (b) 3:2 (v/v) $\mathrm{CCl}_{4}-\mathrm{CH}_{3} \mathrm{CN}$, and (c) $\mathrm{CH}_{3} \mathrm{CN}$ solution of FL $\left(2 \times 10^{-3} \mathrm{M}\right)-\mathrm{TMB}\left(1 \times 10^{-1} \mathrm{M}\right)$ are shown in Figure 1a, b, and $\mathrm{c}$, respectively. The excitation wavelength at $355 \mathrm{~nm}$ corresponds to the ground-state absorption maximum of FL. The ground-state absorption spectra of FL-TMB mixture in two different solvent polarity and individual systems are shown in Figure 2. The ground-state absorption maximum of FL appears at $337 \mathrm{~nm}$. The absorption profile of FL-TMB mixture shows the charge-transfer transition absorption band of ground-state electron donor-acceptor (EDA) complex at $442 \mathrm{~nm}$ and the charge-transfer absorbance increases with solvent polarity. Deconvolution of the absorption profile shows that, at $355 \mathrm{~nm}$, absorption from ground-state EDA complex is much less. Therefore, pump excitation at $355 \mathrm{~nm}$ will preferably excite FL in an FL-TMB mixture.

The possible photoinduced processes that are expected upon excitation are electron-transfer reaction in the singlet electronic manifold, which may lead to the formation of singlet ion pair, and intersystem crossing from the singlet state of the acceptor to its triplet state. It is known from picosecond transient absorption studies that the singlet ion pair between chloranil and aromatic donors is very short-lived, and it reverts back to

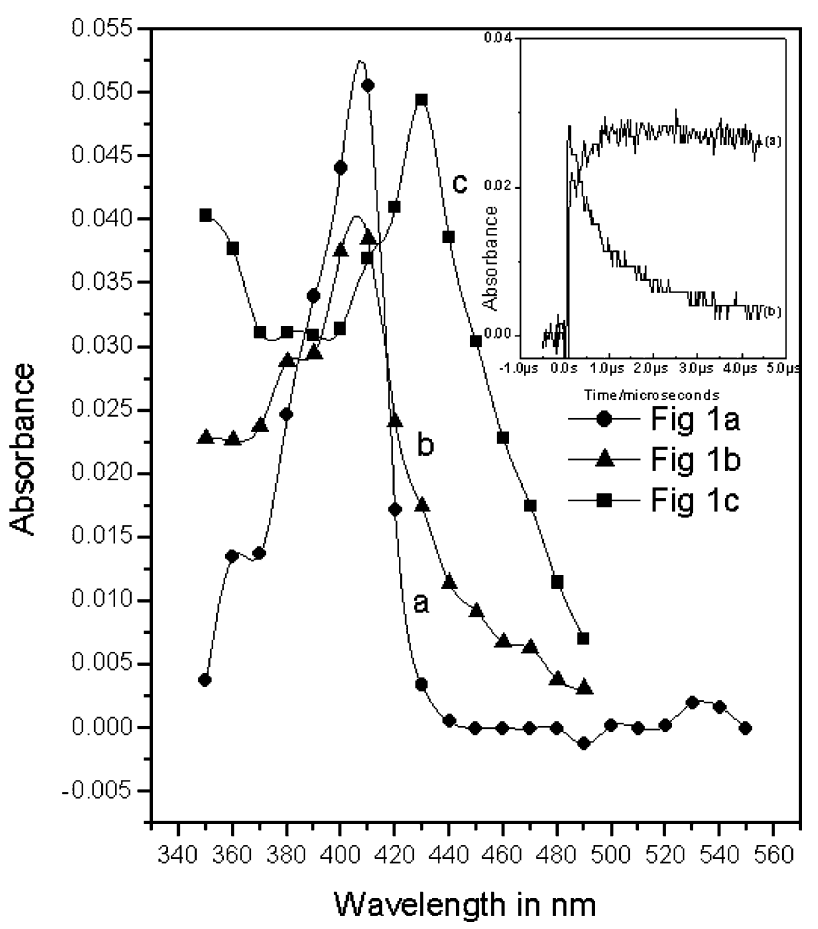

Figure 1. Transient absorption spectra of FL $\left(2 \times 10^{-3} \mathrm{M}\right)$ and TMB $\left(1 \times 10^{-1}\right)$ in (a) 4:1 (v/v) $\mathrm{CCl}_{4}-\mathrm{CH}_{3} \mathrm{CN}$, (b) $3: 2(\mathrm{v} / \mathrm{v}) \mathrm{CCl}_{4}-\mathrm{CH}_{3} \mathrm{CN}$, (c) $\mathrm{CH}_{3} \mathrm{CN}$. Pump laser $355 \mathrm{~nm}$. The spectra are obtained at delay time of $1.0 \mu \mathrm{s}$ after the start of the laser pulse. Inset: (a) Rise of FLH'. Absorption at $410 \mathrm{~nm}$. (b) Decay of the 440-nm absorption of FL $(2 \times$ $\left.10^{-3} \mathrm{M}\right)$ and TMB $\left(1 \times 10^{-1}\right)$ system in $3: 2(\mathrm{v} / \mathrm{v}) \mathrm{CCl}_{4}-\mathrm{CH}_{3} \mathrm{CN}$.

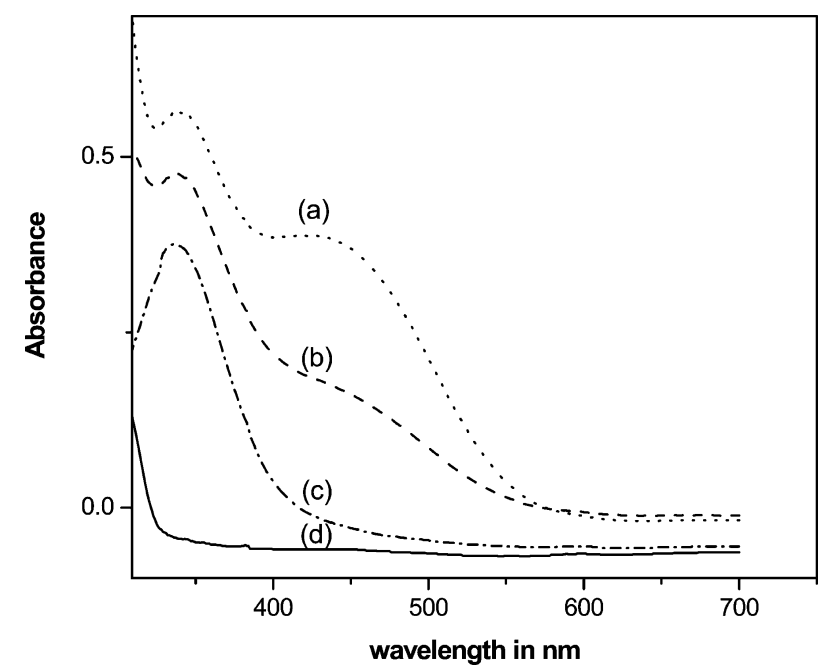

Figure 2. Ground-state absorption spectra of (a) FL $\left(2 \times 10^{-3} \mathrm{M}\right)$ and TMB $\left(1 \times 10^{-1} \mathrm{M}\right)$ in 4:1 (v/v) $\mathrm{CCl}_{4}-\mathrm{CH}_{3} \mathrm{CN}$, (b) $\mathrm{FL}\left(2 \times 10^{-3}\right.$ $\mathrm{M})$ and TMB $\left(1 \times 10^{-1} \mathrm{M}\right)$ in $\mathrm{CH}_{3} \mathrm{CN}$, (c) $\mathrm{FL}\left(2 \times 10^{-3} \mathrm{M}\right)$ in $\mathrm{CH}_{3} \mathrm{CN}$, and $(\mathrm{d}) \mathrm{TMB}\left(1 \times 10^{-1} \mathrm{M}\right)$ in $\mathrm{CH}_{3} \mathrm{CN}$.

the ground state within $10 \mathrm{ps} .{ }^{19}$ Therefore, observation of photoinduced electron-transfer process in the singlet state is not possible with the time resolution of our present experimental setup.

The overall electron-transfer reactivity (singlet vs triplet) will depend on the relative rates of intersystem crossing and electron transfer. The intersystem crossing rates of fluoranil and chloranil are $0.9 \times 10^{11}$ and $1.0 \times 10^{11} \mathrm{~s}^{-1}$, respectively. At low concentration of the donor, electron-transfer reaction proceeds by diffusional encounter between excited state acceptor and donor, and the timescale of diffusional encounter is greater than $1 \mathrm{~ns}$. Therefore, fast ISC from the singlet state of acceptor is 
more favorable compared to the electron-transfer process in the singlet state. Thus, in the present study the photoinduced electron-transfer reaction in the triplet electronic manifold was investigated.

The transient species generated by photoinduced reaction between ${ }^{3} \mathrm{FL}$ and $\mathrm{TMB}$ in $\mathrm{CH}_{3} \mathrm{CN}$ generates a transient absorption band at $430 \mathrm{~nm}$, which is the characteristic of fluoranil radical anion $\left(\mathrm{FL}^{\cdot-}\right){ }^{23}$ Therefore, it is inferred that electron-transfer reaction occurs in $\mathrm{CH}_{3} \mathrm{CN}$. In nonpolar solvent (4:1 (v/v) $\left.\mathrm{CCl}_{4}-\mathrm{CH}_{3} \mathrm{CN}\right)$, the spectra show transient absorption maximum around $410 \mathrm{~nm}$. The absorption band at $410 \mathrm{~nm}$ disappears gradually with a long lifetime compared to the transient generated in $\mathrm{CH}_{3} \mathrm{CN}$. The shift in the absorption maximum of $\left(4: 1(\mathrm{v} / \mathrm{v}) \mathrm{CCl}_{4}-\mathrm{CH}_{3} \mathrm{CN}\right)$ with respect to radical anion of fluoranil is almost the same as those reported ${ }^{23}$ for ketyl radical of $\mathrm{FL}\left(\mathrm{FLH}^{\circ}\right)$; thus, absorption at $410 \mathrm{~nm}$ was assigned to $\mathrm{FLH}^{\circ}$. Figure 1 shows the transient absorption spectra obtained at $1 \mu \mathrm{s}$ in different solvent polarity. It is quite interesting that, in nonpolar media, we do not observe bands of $\mathrm{FL}^{\cdot-}$. In solvents of medium polarity $\left(3: 2(\mathrm{v} / \mathrm{v}) \mathrm{CCl}_{4}-\mathrm{CH}_{3} \mathrm{CN}\right)$, the $410-\mathrm{nm}$ absorption decays at slower rate as compared to that at $430 \mathrm{~nm}$. Therefore, it is inferred that in solvents of medium polarity the spectra consist of absorption bands from both $\mathrm{FL}^{\circ-}$ and $\mathrm{FLH}^{\circ}$. The above results clearly suggest that the competition, between the electron-transfer and the hydrogentransfer reaction, is influenced by the solvent polarity.

The effect of solvent polarity on the spontaneity of ion-pair formation was evaluated from the free energy change $\Delta G$ associated with its formation. In the electron-transfer process, the primary ion-pair intermediate formed in less polar solvents is mainly CIP, whereas SSIP is favored in highly polar media. Thus, in nonpolar media for $4: 1\left(\mathrm{CCl}_{4}-\mathrm{CH}_{3} \mathrm{CN}\right.$ ) (dielectric constant is 13.4) the $\Delta G$ value for the contact ion-pair formation was estimated approximately by eq $1^{10,20}$ to be $-0.562 \mathrm{eV}$, and with the help of eq $2 \Delta G$ for SSIP formation in acetonitrile was estimated to be $-0.621 \mathrm{eV}$. The $\Delta G$ values calculated for both the ion pairs were negative, and therefore ion-pair formation by electron-transfer reaction is feasible in both polar and nonpolar media

$$
\begin{aligned}
& \Delta G\left(\mathrm{FL}^{\bullet-}-\mathrm{TMB}^{\bullet+}\right)_{\mathrm{s}}=E\left(\mathrm{TMB}^{-\mathrm{TMB}^{\bullet+}}\right)- \\
& E\left(\mathrm{FL}^{\bullet-} / \mathrm{FL}\right)-E_{\mathrm{T}}\left({ }^{3} \mathrm{FL}\right)+0.32-0.7(\varepsilon-1 / 2 \varepsilon+1)
\end{aligned}
$$

$$
\begin{aligned}
\Delta G\left(\mathrm{FL}_{\mathrm{s}}^{\bullet-}-\mathrm{TMB}_{\mathrm{s}}^{\bullet+}\right)=E\left(\mathrm{TMB}^{+} \mathrm{TMB}^{\bullet+}\right)- \\
E\left(\mathrm{FL}^{\bullet-} / \mathrm{FL}\right)-E_{\mathrm{T}}\left({ }^{3} \mathrm{FL}\right)-e^{2} / \varepsilon R
\end{aligned}
$$

The dielectric constant of mixed solvent was assumed to be proportional to the mole fractions of those original solvents. $E\left(\mathrm{TMB}^{\mathrm{T}} \mathrm{TMB}^{\cdot+}\right)$ and $E\left(\mathrm{FL}^{\cdot-} / \mathrm{FL}\right)$ are the oxidation potential of TMB $(1.59 \mathrm{~V})$ and the reduction potential of FL $(-0.04 \mathrm{~V})$, respectively, in acetonitrile. The energy of ${ }^{3} \mathrm{FL}\left(E_{\mathrm{T}}\left({ }^{3} \mathrm{FL}\right)\right)$ is 2.2 $\mathrm{eV},{ }^{21} \varepsilon$ is the dielectric constant, and $R$ is the distance, typically $7.5 \AA$, between the donor and the acceptor for a loose ion pair. ${ }^{16}$

Free energy calculations show that ion-pair formation is feasible in both polar and nonpolar media, whereas transient absorption spectra obtained in nonpolar media show the presence of only $\mathrm{FLH}^{*}$. The possible reason for this could be that the hydrogen-transfer sites in the collision complex between ${ }^{3} \mathrm{FL}$ and TMB are present in close proximity in nonpolar media, which favors hydrogen-transfer reaction to proceed with a high frequency factor. The collision complex might result from the formation of an initial hydrogen-bonding bridge between $\mathrm{O}$-atom of the acceptor and $\mathrm{H}$-atom of the methyl group on the donor. A fast hydrogen transfer by a two-step mechanism of electron transfer followed by proton transfer has been reported in electron donor-acceptor and hydrogen-bonding interaction systems. ${ }^{20}$ Taking this reported observation into account, it is also possible that the formation of $\mathrm{FLH}^{\bullet}$ in nonpolar solvents might also proceed through a two-step mechanism of fast electron transfer followed by proton transfer.

In polar solvents, proton transfer in the ion pair may be restricted, whereas in medium polar solvents the ion pairs are likely to undergo proton-transfer reaction. In such a case, the decay of the ion-pair absorption in medium polarity will be accompanied by growth of the transient absorption from $\mathrm{FLH}^{\circ}$. This has been analyzed from the temporal change in the transient absorption at $410 \mathrm{~nm}$, which is characteristic of FLH. The pure $\mathrm{FL}^{--}$spectrum free from $\mathrm{FLH}^{\bullet}$ (Figure 1c) (obtained in polar solvent) has a considerable amount of absorption at $410 \mathrm{~nm}$ due to $\mathrm{FL}^{\circ-}$. Therefore, the temporal change in transient absorption at $410 \mathrm{~nm}$ obtained in medium polar media involves absorption from both $\mathrm{FL}^{--}$and $\mathrm{FLH}^{\circ}$. With the help of eq 3, the contribution from radical anion absorption was subtracted from the net spectrum obtained at $410 \mathrm{~nm}$ in medium polarity.

$$
{ }^{\mathrm{N}} A={ }^{\mathrm{M}} A_{410}-\left({ }^{\mathrm{P}} A_{410} /{ }^{\mathrm{P}} A_{440}\right) \times{ }^{\mathrm{M}} A_{440}
$$

${ }^{\mathrm{N}} A$ is the subtracted spectrum. ${ }^{\mathrm{M}} A_{410}$ and ${ }^{\mathrm{M}} A_{440}$ are the absorbance at 410 and $440 \mathrm{~nm}$, respectively, obtained in medium polar media. ${ }^{\mathrm{P}} A_{410}$ and ${ }^{\mathrm{P}} A_{440}$ are the absorption at 410 and 440 $\mathrm{nm}$, respectively, obtained in polar media. The use of transient absorption decay profile at $440 \mathrm{~nm}$ is necessary since at this wavelength absorption due to ketyl radical is negligible. The temporal change in the transient absorption at $410 \mathrm{~nm}$ obtained after subtracting the contribution from $\mathrm{FL}^{-}$is shown as inset in Figure 1 along with the transient decay at $440 \mathrm{~nm}$ corresponding to $\mathrm{FL}^{\cdot-}$ absorption. It shows temporal growth in 410$\mathrm{nm}$ absorption. In contrast, the temporal profile of $\mathrm{FLH}^{\bullet}$ absorption in nonpolar solvent does not show rise in the transient absorption. Therefore, it is inferred that intra-ion-pair proton transfer occurs in medium polar solvent and fast hydrogentransfer process takes place in nonpolar solvent. In the Figure 1 inset, the rise time ( $310 \mathrm{~ns}$ ) of the $410-\mathrm{nm}$ band due to $\mathrm{FLH}^{*}$ absorption and the first-order decay time (900 ns) of the 440$\mathrm{nm}$ band due to $\mathrm{FL}^{\cdot-}$ absorption do not match properly. The possible reason could be that $\mathrm{FLH}^{\bullet}$ generated by collisional encounter between ${ }^{3} \mathrm{FL}$ and TMB might also contribute to the temporal profile of the 410-nm absorption. While the major contribution of the absorption at $440 \mathrm{~nm}$ is from CIP, it will also have some amount of contribution from long-lived solvated ions resulting from the dissociation of SSIP, and this will also lead to mismatch between the decay time of ion pair and rise of $\mathrm{FLH}^{\circ}$.

The CIP, which has some amount of mixing between the ionic and the locally excited triplet complex, is well-known as exciplex. The exciplex nature of the CIP will lead to structural difference between the CIP and SSIP. Time-resolved resonance Raman studies can provide better information regarding the nature of ion pair formed, because the extent of charge transfer in the ion pair, which differentiates the CIP from SSIP, can be observed in the time-resolved vibrational spectra.

Time-Resolved Resonance Raman Study. TR3 studies on photoinduced electron-transfer reaction between ${ }^{3} \mathrm{FL}$ and TMB were conducted at pump wavelength $355 \mathrm{~nm}$ and probe wavelength $416 \mathrm{~nm}$ in different mixtures of $\mathrm{CH}_{3} \mathrm{CN}$ and $\mathrm{CCl}_{4}$. 


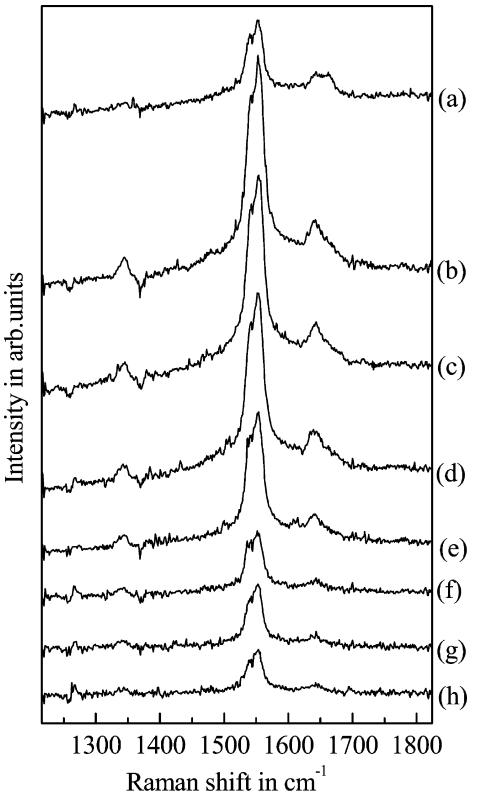

Figure 3. Time-resolved resonance Raman spectra of FL $\left(2 \times 10^{-3}\right.$ $\mathrm{M})$ and TMB $\left(1 \times 10^{-1} \mathrm{M}\right)$ in $4: 1(\mathrm{v} / \mathrm{v}) \mathrm{CCl}_{4}-\mathrm{CH}_{3} \mathrm{CN}$ obtained at various delay times (pump laser $355 \mathrm{~nm}$, probe laser $416 \mathrm{~nm}$ ). (a) 0 ns, (b) $68 \mathrm{~ns}$, (c) $168 \mathrm{~ns}$, (d) $368 \mathrm{~ns}$, (e) $1.3 \mu \mathrm{s}$, (f) $5.3 \mu \mathrm{s}$, (g) $9.3 \mu \mathrm{s}$, (h) $17.3 \mu \mathrm{s}$. Pump-only and probe-only spectra were subtracted from the pump+probe spectra.

The transient absorption spectra showed that both $\mathrm{FL}^{--}$and $\mathrm{FLH}^{\circ}$ sufficiently absorb at $416 \mathrm{~nm}$. Therefore, at this probe wavelength resonance enhancement due to both the species can occur.

TR3 spectra obtained in 4:1 (v/v) $\mathrm{CCl}_{4}-\mathrm{CH}_{3} \mathrm{CN}$ mixture at various delays are shown in Figure 3. It consists of an intense band at $1550 \mathrm{~cm}^{-1}$ and a weak band at $1638 \mathrm{~cm}^{-1}$, which resemble the resonance Raman spectra of $\mathrm{FLH}^{\circ}{ }^{14}$ At 0 -ns delay between the pump and probe, a weak shoulder band at 1662 $\mathrm{cm}^{-1}$ is noticed in the TR3 spectra, and at a later time (after 65 ns) this band decays. The weak shoulder band could be due to the presence of the ion pair (vide infra). Transient absorption results showed that the ion-pair formation is less favorable in nonpolar solvents. The TR3 spectra consisting of a vibrational band predominantly from $\mathrm{FLH}^{*}$ are consistent with the transient absorption studies.

The TR3 spectra obtained in $\mathrm{CH}_{3} \mathrm{CN}$ at various delays between the pump and probe are shown in Figure 4. The TR3 spectra at initial (within $240 \mathrm{~ns}$ ) are similar to the $\mathrm{FL}^{-}$. The resemblance of the TR3 spectra of $\mathrm{FL}-\mathrm{TMB}$ in $\mathrm{CH}_{3} \mathrm{CN}$ with the resonance Raman spectrum of $\mathrm{FL}^{-16}$ confirms that the early time TR3 spectra are due to $\mathrm{FL}^{--}$. The band at $1667 \mathrm{~cm}^{-1}$ decays at a faster rate compared to the one at $1560 \mathrm{~cm}^{-1}$. This is due to the presence of ketyl radical whose TR3 vibrational frequencies are very close to that of the radical anion but the relative intensities are just reversed. ${ }^{22}$ At later time (after $2.1 \mu \mathrm{s}$ ), the presence of a very weak band due to $\mathrm{FLH}^{\circ}$ is observed in the TR3 spectra. The major content in the spectra is TR3 bands of $\mathrm{FL}^{\cdot-}$. Occurrence of electron-transfer reaction in polar solvents is consistent with the transient absorption results.

The TR3 spectra at various delays obtained in solvents of medium polarity containing $3: 2(\mathrm{v} / \mathrm{v}) \mathrm{CCl}_{4}-\mathrm{CH}_{3} \mathrm{CN}$ mixture are shown in Figure 5. The early time (below 26 ns) shows two bands at 1667 and $1560 \mathrm{~cm}^{-1}$. The relative intensity of both the bands at early time is different from those obtained in polar and nonpolar media. This shows that the extent of radical anion formation is much higher compared to those observed in nonpolar solvents, and the amount of ketyl radical formation is

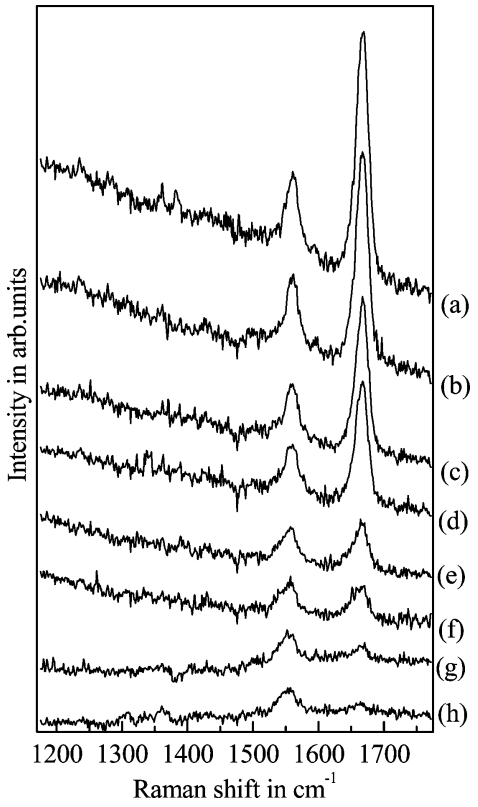

Figure 4. Time-resolved resonance Raman spectra of FL $\left(2 \times 10^{-3}\right.$ $\mathrm{M})$ and TMB $\left(1 \times 10^{-1}\right)$ in $\mathrm{CH}_{3} \mathrm{CN}$ obtained at various delay times (pump laser $355 \mathrm{~nm}$, probe laser $416 \mathrm{~nm}$ ). (a) $10 \mathrm{~ns}$, (b) $40 \mathrm{~ns}$, (c) 140 ns, (d) $240 \mathrm{~ns}$, (e) $1.2 \mu \mathrm{s}$, (f) $2.1 \mu \mathrm{s}$, (g) $4.1 \mu \mathrm{s}$, (h) $6.1 \mu \mathrm{s}$. Pump-only and probe-only spectra were subtracted from the pump + probe spectra.

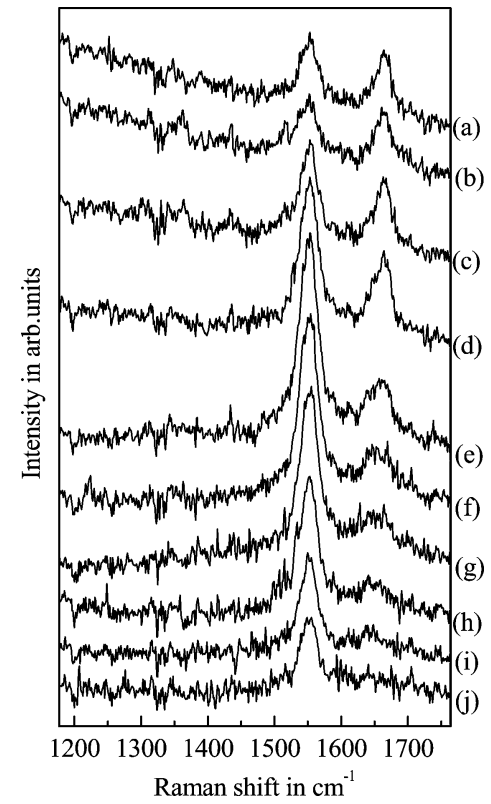

Figure 5. Time-resolved resonance Raman spectra of FL $\left(2 \times 10^{-3}\right.$ $\mathrm{M})$ and TMB $\left(1 \times 10^{-1}\right)$ in $3: 2(\mathrm{v} / \mathrm{v}) \mathrm{CCl}_{4}-\mathrm{CH}_{3} \mathrm{CN}$ obtained at various delay times (pump laser $355 \mathrm{~nm}$, probe laser $416 \mathrm{~nm}$ ). (a) $10 \mathrm{~ns}$, (b) $12 \mathrm{~ns}$, (c) $16 \mathrm{~ns}$, (d) $26 \mathrm{~ns}$, (e) $66 \mu \mathrm{s}$, (f) $166 \mathrm{~ns}$, (g) $266 \mathrm{~ns}$, (h) $1.2 \mu \mathrm{s}$, (i) $3.2 \mu \mathrm{s}$, (j) $5.2 \mu \mathrm{s}$. Pump-only and probe-only spectra were subtracted from the pump+probe spectra.

much higher compared to those observed in polar solvents. In the process of decay, the intense radical anion band at 1667 $\mathrm{cm}^{-1}$ decays much faster and the presence of long-lived bands of ketyl radical is observed in the spectra.

The nature of the ion pair in different solvent polarity was investigated from the changes observed in the vibrational frequency of the FL part of the complex. In medium polar solvent, if the CIP is a triplet exciplex then the vibrational frequency will be shifted from the pure radical anion frequency toward the corresponding mode of the triplet state. In polar 


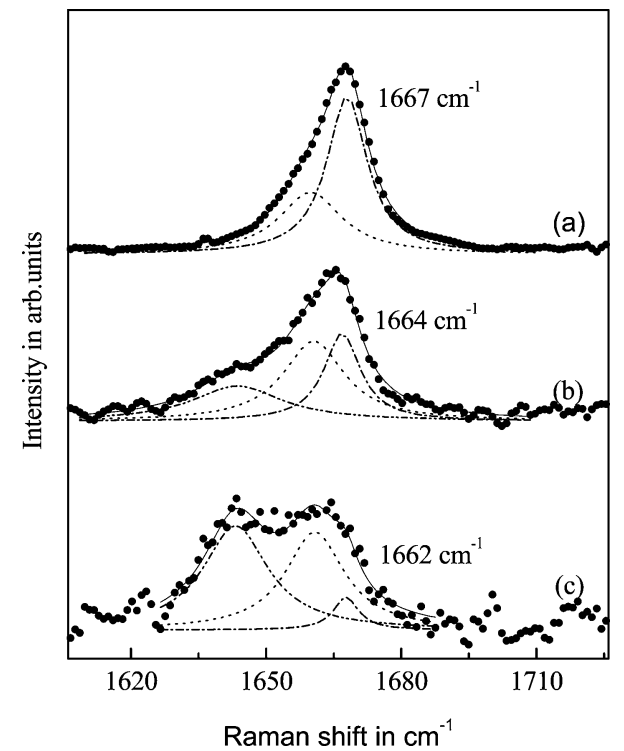

Figure 6. Time-resolved resonance Raman spectra of FL $\left(2 \times 10^{-3}\right.$ M) and TMB $\left(1 \times 10^{-1}\right)($ pump laser $355 \mathrm{~nm}$, probe laser $416 \mathrm{~nm})$ in (a) $\mathrm{CH}_{3} \mathrm{CN}$ at $20 \mathrm{~ns}$, (b) $3: 2(\mathrm{v} / \mathrm{v}) \mathrm{CCl}_{4}-\mathrm{CH}_{3} \mathrm{CN}$ at $20 \mathrm{~ns}$, and (c) $4: 1$ (v/v) $\mathrm{CCl}_{4}-\mathrm{CH}_{3} \mathrm{CN}$ at $0 \mathrm{~ns}$. Pump-only and probe-only spectra were subtracted from the pump + probe spectra.

solvents, the SSIP will be closer to the vibrational frequency of the radical anion. TR3 studies carried out by Tahara and Hamaguchi ${ }^{17}$ on chloranil alkylbenzene triplet charge-transfer complex made a similar observation.

The change in the vibrational frequency due to the difference in the nature of the ion pair was observed in the case of the most intense band of $\mathrm{FL}^{--}$at $1667 \mathrm{~cm}^{-1}$, which corresponds to $\mathrm{C}=\mathrm{C}$ stretching frequency. The corresponding mode of ${ }^{3} \mathrm{FL}$ reported in acetonitrile is at $1603 \mathrm{~cm}^{-1}$. Therefore, if the CIP is a triplet exciplex then due to mixing between the triplet locally excited state and the charge-transfer state, the band at $1667 \mathrm{~cm}^{-1}$ in the CIP should get shifted toward lower frequency. Figure 6 shows the TR3 band at $1667 \mathrm{~cm}^{-1}$ due to ion pair in different solvent polarity. In polar solvent TR3 band due to the ion pair consists of two bands observable from its unsymmetrical band shape. In medium and nonpolar solvents, the less intense band due to ketyl radical is also present along with the ion-pair band. The ion-pair band at $1667 \mathrm{~cm}^{-1}$ in polar solvents shifts to 1664 $\mathrm{cm}^{-1}$ in medium polar solvent. In nonpolar solvent, it gets further shifted to $1662 \mathrm{~cm}^{-1}$. The asymmetrical shape of the $1667 \mathrm{~cm}^{-1}$ band in polar solvent and its gradual shift (toward lower frequency) with decrease in solvent polarity suggest that the TR3 bands of ion pair consist of bands from two different species, one at lower and the other at slightly higher vibrational wavenumber. To identify the intermediates, we analyzed the change in intensity of the two intermediate species in each medium by carrying out a deconvolution of the Raman bands. Band fitting analyses were carried out with the assumption of a Lorentzian line shape of the Raman bands. The ion-pair band in polar solvent was fitted to two Lorentzian line shapes, whereas due to the presence of TR3 bands from the ketyl radical the Raman bands in medium and nonpolar solvent were fitted to three (based on the observed band contours) Lorentzian line shapes. The resulting spectra are shown in Figure 6. The deconvolution results in two bands for the ion-pair one at 1667 $\mathrm{cm}^{-1}$ and the other at $1660 \mathrm{~cm}^{-1}$. With decrease in solvent polarity, the intensity of the $1667 \mathrm{~cm}^{-1}$ band decreases and the $1660 \mathrm{~cm}^{-1}$ band increases. With respect to the $1667 \mathrm{~cm}^{-1}$ vibrational band, the $1660 \mathrm{~cm}^{-1}$ band is shifted toward the

\section{SCHEME 2}

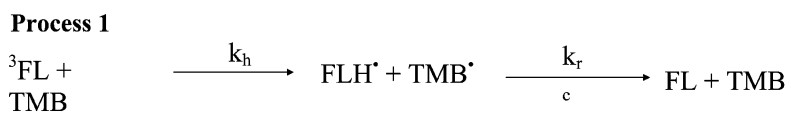

Process 2

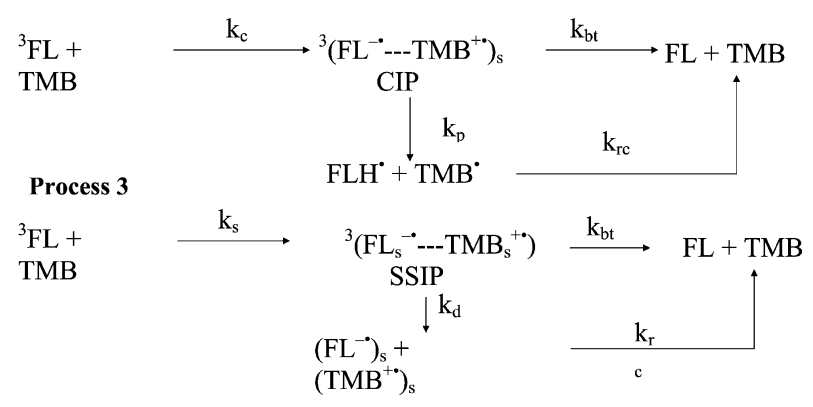

corresponding vibrational mode of the triplet state, and moreover it is more intense for the ion-pair band in less polar media. Therefore, it is suggested that the $1660 \mathrm{~cm}^{-1}$ is a CIP and it is a triplet exciplex in nature, whereas the $1667 \mathrm{~cm}^{-1}$ band is very intense in polar media and therefore it corresponds to SSIP. In SSIP, the ion pairs are separated by solvents, and therefore structurally they cannot be differentiated from the solvated ions. Therefore, the band, which appears at higher wavenumbers, is due to the presence of both SSIP and solvated ions. The exciplex or CIP is stabilized by Coulombic interaction and by solvation. The stabilization gained by solvation is favored by increasing the solvent polarity. In highly polar solvents, the ion pairs get solvated rapidly, and in the process of solvation the solvent molecules penetrate the space between the ions, which results in screening of the Coulombic attraction between the ions. Therefore, in highly polar solvent, stabilization is gained because of the solvation of the ions $\left(\Delta G_{\mathrm{sol}}\right)$, and the nature of ion pair is SSIP.

From the results obtained from transient absorption and TR3 studies, the effect of solvent polarity on the mechanism of photoinduced electron-transfer reaction between ${ }^{3} \mathrm{FL}$ and TMB has been outlined in Scheme 2. The subscripts $\mathrm{c}$ and s refer to the processes of CIP and SSIP formation, respectively, and h, $\mathrm{p}, \mathrm{d}, \mathrm{bt}$, and rc refer in turn to hydrogen transfer, proton transfer, ionic dissociation, back electron transfer, and recombination, respectively. In nonpolar solvent, process 1 is mostly favored, in medium polar media both processes 1 and 2 take place, whereas in polar media process 3 is the major reaction pathway.

The effect of solvent polarity on the mechanism of photoinduced electron-transfer reaction in the case of FL is quite different from that of $p$-chloranil. ${ }^{9,10,17}$ Transient absorption and TR3 studies on photoinduced electron-transfer reaction between chloranil and aromatic donors suggest that in nonpolar solvents such as 1,2-dichloroethane (dielectric constant of 10.3) 80\% of the collison undergoes electron-transfer reaction to form the ion pair, whereas $20 \%$ of the collison complex undergoes H-transfer reaction. ${ }^{10}$ In contrast to this, in our present study the effect of nonpolar media in $4: 1(\mathrm{v} / \mathrm{v}) \mathrm{CCl}_{4}-\mathrm{CH}_{3} \mathrm{CN}$ (dielectric constant $=13.4$ ) results in formation of ketyl radical without the presence of ion-pair intermediate even though the polarity is slightly higher than that of 1,2-dichloroethane. The results clearly show that the perfluoro effect imparted by fluorine substitution results in difference in reactivity of FL compared to $p$-chloranil.

\section{Conclusions}

In this work, the effect of solvent polarity on the mechanism and the nature of ion-pair intermediates formed in photoinduced 
electron-transfer process was investigated using transient absorption and TR3 spectroscopy. Hydrogen transfer was found to compete with the electron-transfer mechanism. In nonpolar media, the formation of ketyl radical due to hydrogen transfer from TMB to FL is favored. As the polarity of the solvent is increased, the hydrogen-transfer mechanism switches over to electron-transfer mechanism. The nature of primary ion-pair intermediates generated in different solvent polarity was investigated from the structural changes in the acceptor following charge transfer in the ion pair. In medium polar media, contact ion pair of exciplex nature is more favored, and this species eventually decays via intra-ion-pair proton transfer. In polar solvent, complete ionic nature of the solvent-separated ion-pair was observed.

Acknowledgment. We thank Dr. P. Ramamurthy for useful discussion and the National Centre for Ultrafast Processes, Chennai, for providing nanosecond transient absorption facilities. We thank the National Laser Programme of the Department of Science and Technology (DST) and the Council of Scientific and Industrial Research (CSIR) for financial support.

\section{References and Notes} 791.

(1) Leonhardt, H.; Weller, A.; Bunsenges, B. Phys. Chem. 1963, 67,

(2) Beens, H.; Weller, A. In Organic Molecular Photophysics; Birks, J. B., Ed.; Wiley: London, 1975; Vol. 2, Chapter 4.

(3) Masuhura, H.; Mataga, N. Acc. Chem. Res. 1981, 14, 312.

(4) Mataga, N. Pure. Appl. Chem. 1984, 56, 1255.
(5) Mattes, S. L.; Farid, S. J. Chem. Soc., Chem. Commun. 1980, 128.

(6) Mattes, S. L.; Farid, S. J. Am. Chem. Soc. 1983, 105, 1386.

(7) Mattes, S. L.; Farid, S. J. Am. Chem. Soc. 1986, 108, 7356.

(8) Gould, I. R.; Young, R. H.; Moody, R. E.; Farid, S. J. Phys. Chem. 1991, 95, 2068.

(9) Kobashi, H.; Okabe, S.; Ohsugi, Y.; Shizuka, H. Bull. Chem. Soc. Jpn. 1990, 63, 2173.

(10) Kobashi, H.; Funabashi, M.; Kondo, T.; Morita, T.; Okada, T.; Mataga, N. Bull. Chem. Soc. Jpn. 1984, 57, 3557.

(11) Kobashi, H.; Suto, H.; Sizuka, H. Bull. Chem. Soc. Jpn. 1990, 63, 1441.

(12) Chowdhury, S.; Grimsrud, E. P.; Heinis, T.; Kebarle, P. J. Am. Chem. Soc. 1986, 108, 3635.

(13) Balakrishnan, G.; Umapathy, S. J. Chem. Soc., Faraday Trans. 1997, 93, 4125 .

(14) Balakrishnan, G.; Umapathy, S. Chem. Phys. Lett. 1997, $270,557$.

(15) Vauthey, E.; Phillips, D.; Parker, A. W. J. Phys. Chem. 1992, 96, 7356.

(16) Vauthey, E.; Parker, A. W.; Nohova, B.; Phillips, D. J. Am. Chem. Soc. 1994, 116, 9182.

(17) Tahara, T.; Hamaguchi, H. J. Phys. Chem. 1992, 96, 8252.

(18) Balakrishnan, G.; Mohandas, P.; Umapathy, S. J. Phys. Chem. 1996, $100,16472$.

(19) Hubig, S. M.; Bockman, T. M.; Kochi, J. K. J. Am. Chem. Soc. 1997, 119, 2926.

(20) (a) Ikeda, N.; Okada, T.; Mataga, N. Bull. Chem. Soc. Jpn. 1981, 54, 1025. (b) Weller, A.; Zachariasse, K. In Molecular Luminescence; Lim, E. C., Ed.; W. A. Benjamin: New York, 1969; p 895. (c) WellerA. In The Exciplex; Gordon, M., Ware, W. R., Eds.; Academic Press: New York, 1975; p 23.

(21) Darmanyan, A. P.; Foote, C. S. J. Phys. Chem. 1992, 96, 6317.

(22) Shoute, L. C. T.; Mittal, J. P. J. Phys. Chem. 1994, 98, 11094.

(23) Balakrishnan, G.; Mohandas, P.; Umapathy, S. J. Phys. Chem A 2001, 105, 7778 .

JP903973Q 